\title{
Tele-Audiological Surveillance of Middle Ear Status among Individuals with Cleft Lip and/or Palate in Rural South India
}

DOI: $10.3766 /$ jaaa. 18085

\author{
Pavithra Ravi* \\ Vidya Ramkumar* \\ Akilan Rajendran* \\ Prasanna Kumar Saravanam $\dagger$ \\ Subramaniyan Balasubramaniyan* \\ Roopa Nagarajan*
}

\begin{abstract}
Background: A community-based program for the comprehensive management of communication disorders among individuals with cleft lip and/or palate (CLP) was implemented in two rural districts in the state of Tamil Nadu, India. The program was successful in terms of early surgical repair, orthodontic management and speech correction; however, audiological surveillance was challenging due to poor follow-up rates.
\end{abstract}

Purpose: The aim of the study was to compare tele-audiological and in-person audiological surveillance to identify the most beneficial approach for the identification and treatment of middle ear disease (MED) in individuals with CLP in this rural community-based program.

Research Design: Quasi-experimental study.

Study Sample: All beneficiaries older than 5 years actively seeking services in Cuddalore $(n=44)$ and Thiruvannamalai $(n=65)$ districts of Tamil Nadu, India, were included in the study. Individuals in Cuddalore district were assigned to the tele-audiological surveillance group, whereas individuals in Thiruvannamalai district were assigned to the in-person audiological surveillance group.

\begin{abstract}
Methods: In the tele-audiological surveillance group, video-otoscopy examinations were performed by trained community-based rehabilitation workers (CBRWs). Pure-tone audiometry and tympanometry were performed by an audiologist from the tertiary care hospital through remotely accessed equipment using broadband internet at the participants' homes and monthly camps. Findings from those with suspected MED were forwarded to the otolaryngologist at the tertiary care hospital for remote diagnoses and treatment plans. Subsequently, CBRWs relayed the otolaryngologist's recommendations to the individuals receiving services at the community. In the in-person audiological surveillance group, video-otoscopy, pure-tone audiometry, and tympanometry were performed by the audiologist following standard testing procedures at monthly camps. Individuals with suspected MED and impacted cerumen were referred for inperson diagnoses and treatment plans. Follow-up audiological surveillance was conducted in both districts after implementation of the otolaryngologist's recommendations.
\end{abstract}

Data Analysis: The coverage, follow-up rate for intervention and cost-outcomes from the provider's perspective were analyzed and compared between two groups.

Results: Tele-audiological surveillance provided superior coverage (68\%) compared with in-person audiological surveillance (38\%). The tele-audiological surveillance group showed greater follow-up compliance

*Department of Speech, Language and Hearing Sciences, Sri Ramachandra Institute for Higher Education and Research (Deemed to be University), Chennai, India; †Department of ENT, Head and Neck Surgery, Sri Ramachandra Institute for Higher Education and Research (Deemed to be University), Chennai, India

Corresponding author: Vidya Ramkumar, Department of Speech, Language and Hearing Sciences, Sri Ramachandra Institute for Higher Education and Research (Deemed to be University), Chennai, India 600116; Email: vidya.ramkumar@sriramachandra.edu.in

We thank Transforming Faces, Canada for their funding support and Medtronic India Pvt. Ltd for equipment support. 
$(61 \%)$ than the in-person audiological surveillance group (19\%) for treatment plans such as cerumen management and hearing aid fitting provided locally. Compared with in-person audiological surveillance, tele-audiological surveillance resulted in cost saving of USD 47 per individual.

Conclusion: Tele-audiological surveillance was beneficial for the purpose of identifying and providing treatment in patients having CLP with MEDs in rural locations.

Key Words: audiological surveillance, cleft, community-based, community-based rehabilitation worker, lip and/or palate, middle ear disease, tele-audiology, video-otoscopy

\begin{abstract}
Abbreviations: $A B R=$ auditory brainstem response; $A C=$ air conduction; $B C=$ bone conduction; CBRW = community-based rehabilitation worker; CLP = cleft lip and palate; MED = middle ear disease; $\mathrm{NGO}=$ nongovernmental organization; SRMC = Sri Ramachandra Institute Medical College; TM = tympanic membrane
\end{abstract}

\section{INTRODUCTION}

I ndividuals with cleft lip and palate (CLP) are at greater risk of middle ear disease (MED) (Subramaniam et al, 2015) because of the lack of patency of the eustachian tube owing to palatal muscle dysfunction (Bluestone et al, 1972; Kuehn and Moller, 2000). Recurrent MED increases the risk of permanent hearing loss in such individuals (Flynn et al, 2009). Early identification of MED in individuals with CLP is necessary to prevent this complication. As facial deformities and speech intelligibility are the primary concerns of parents and caregivers, ear- and hearing health status are often overlooked (Strauss et al, 1988; Millard and Richman, 2001; Subramaniam et al, 2015).

The American Cleft-Palate Craniofacial Association (2009) recommends routine audiological surveillance for individuals with CLP to promote prevention and early identification of MED. A community-based program for the comprehensive management of communication disorders among individuals with CLP was developed by the Sri Ramachandra Institute Medical College (SRMC) and Research Institute (Deemed to be University), in Chennai, India, in 2005. The program targeted individuals with CLP living in Thiruvannamalai and, subsequently, expanded to Cuddalore in 2011, both rural areas in the state of Tamil Nadu in South India. Between 2005 and 2017, 564 beneficiaries were identified by trained, grass-roots level community-based rehabilitation workers (CBRWs). All beneficiaries underwent surgical repair at SRMC's tertiary care hospital. Follow-up services for speech and language assessment and therapy, orthodontic evaluation, dental screening, and audiological screening were provided at monthly community-based camps. Because speech correction services were required on an ongoing basis, trained CBRWs provided therapy for correction of the "error consonant" under the supervision of the speech language pathologist.

The program achieved success in terms of early surgical repair of CLP; however, early orthodontic management, speech correction, and identification and management of ear- and hearing-related disorders were challenging.
During the initial 5-year period of the program, otoscopy, pure-tone audiometry (air conduction [AC]) and tympanometry screenings were conducted using handheld devices (MT 10; Interacoustics, Denmark) at camps. However, camp-based screenings were not effective in achieving the program's goals for audiological surveillance. For example, AC screenings were not robust in identifying conductive components because of the middle ear pathology, bone conduction (BC) testing was not explored as ambient noise levels could not be monitored without appropriate instrumentation, and tympanometry was not sensitive in identifying middle ear problems in individuals with CLP. Feniman et al (2008), reported that only $65 \%$ of infants showed middle ear problems through tympanometry, as compared with 84\% through otomicroscopy. D'Mello and Kumar (2007) reported that tympanometry, with confirmation by the otolaryngologist, was successful in detecting the presence of middle ear effusion, cerumen, and chronic suppurative otitis media.

Camp-based screenings had the disadvantage of poor follow-up compliance rates for diagnoses and treatments performed by otolaryngologists at the tertiary care hospital or local clinics. Otolaryngologists were unable to participate in the monthly camps because of other commitments.

Based on recent advancement in telehealth in providing services, we aimed to explore tele-audiological surveillance methods performed with the assistance of CBRWs. However, the provision of such services needs to be evaluated in comparison with the existing model of in-person testing performed at the campsite to assess its strengths and challenges. Such evaluation can provide insights for future program planning, which may maximize patient outcomes.

A previous study in Australia revealed that the use of tele-audiology to provide hearing services to remote areas had the benefits of improved service provision time and coverage (Pearce et al, 2009). Moreover, tele-audiology showed potential to expand provision of hearing health care by enabling access to services in remote, rural populations (Brennan-Jones et al, 2018) 
In the present study, we aimed to compare the outcomes of tele-audiological surveillance and ongoing camp-based in-person audiological surveillance to identify the most beneficial approach for the identification and treatment of MED in individuals with CLP in two rural districts in the state of Tamil Nadu, India.

\section{METHODS}

\section{Ethics Approval}

The study was approved by the Institutional Ethics Committee of Sri Ramachandra Medical College and Research Institute (Deemed to be University), Porur, Chennai, Tamil Nadu, India. All participants provided written informed consent.

\section{Study Location}

The study was conducted in Cuddalore and Thiruvannamalai districts, located approximately $180 \mathrm{~km}$ from the SRMC tertiary care hospital. A map of Tamil Nadu depicting the two districts under study is shown in Figure 1.

\section{Sampling Frame}

All beneficiaries older than 5 years (in this study, children younger than 5 years were not included as specialized test equipment would have been required for audiological surveillance) actively seeking follow-up services in Cuddalore $(\mathrm{n}=44)$ and Thiruvannamalai $(\mathrm{n}=65)$ districts were included in the audiological surveillance.

Individuals in Cuddalore district were assigned to the tele-audiological surveillance group, as the CBRWs in this district had undergone training in video-otoscopy and were experienced in using the technology, whereas individuals in Thiruvannamalai district were assigned to the in-person audiological surveillance group.

\section{Test Procedures Included in Audiological Surveillance}

Audiological surveillance was conducted using videootoscopy, pure-tone audiometry, and tympanometry.

Video-otoscopy was performed using the Medtronic mobile phone-based video-otoscope (http://www.medtronic. com/in-en/about/shruti.html). The individual's ear- and hearing-related demographic data and case history were entered into an Android mobile application. Subsequently, images of the tympanic membranes (TMs) were captured and uploaded to a remote access site (cloud) using the application. The images were reviewed by an audiologist at the tertiary care hospital (telemode) or at the campsite (in-person mode). When required, the audiologist forwarded images to an otolaryngologist for provisional diagnoses and the
CBRWs communicated the otolaryngologist's recommendations to the beneficiaries.

Audiometry was conducted using the Senteiro Path audiometer (SOD100497; Germany) with TDH 39 headphones (Telephonics ${ }^{\circledR}, \mathrm{NY}$ ) and B-71 bone vibrators (Radioear, Denmark). Air conduction thresholds at 500 $\mathrm{Hz}, 1 \mathrm{kHz}, 2 \mathrm{kHz}, 4 \mathrm{kHz}$, and $8 \mathrm{kHz}$ and $\mathrm{BC}$ thresholds from $500 \mathrm{~Hz}$ to $4 \mathrm{kHz}$ were obtained to estimate the type and degree of hearing loss. Individuals with hearing thresholds $\leq 25 \mathrm{~dB}$ HL were considered to have normal hearing (WHO, 1991).

Tympanometry was conducted using the Sentiero Path immittance audiometer and reflexometry (SOD100497) and reflexometry using the $226-\mathrm{Hz}$ probe tone at peak pressure ranging from -200 to +200 decaPascals. Reflexometry was performed manually at $500 \mathrm{~Hz}, 1 \mathrm{kHz}, 2 \mathrm{kHz}$, and $4 \mathrm{kHz}$ under both ipsilateral and contralateral conditions.

\section{Tele-Audiological Surveillance}

Individuals in Cuddalore district were scheduled for tele-audiological surveillance with the assistance of trained CBRWs. The CBRWs conducted video-otoscopy testing asynchronously (stored and forwarded), and the audiologist conducted pure-tone audiometry and tympanometry synchronously (in real time).

\section{Training CBRWs as Facilitators}

Two audiologists trained the CBRWs to operate a laptop, establish internet connectivity using a mobile hotspot, and connect a microphone and web camera to establish videoconferencing using Team Viewer version 12. The CBRWs were also trained to connect audiological testing equipment to the laptops and place transducers on the individual to be tested.

\section{Test Location and Testing Setup}

Tele-audiological surveillance was scheduled either at campsites (24 individuals) or at the individual's homes (20 individuals), depending on the availability of mobile data network and ambient noise levels of $\leq 40 \mathrm{dBA}$, determined using BOSCH-iV (Android mobile application; Robert Bosch Engineering and Business Solutions Limited, Germany). The CBRWs established internet connections using a $4 \mathrm{G}$ Jio mobile hotspot. Audiologists used a 4G dongle (Vodafone Idea Ltd, India) at the tertiary care hospital to remotely access the test equipment. The average download speed was $11 \mathrm{Mbps}$ (range, 0.55-133.12 Mbps) and upload speed was $16 \mathrm{Mbps}$ (range, 0.11-180.53 Mbps). First, CBRWs conducted video-otoscopy and uploaded images to the cloud. Next, audiologists at remote locations gave instructions for conducting pure-tone audiometry and 
tympanometry. Finally, CBRWs placed transducers for testing the $\mathrm{AC}$ and $\mathrm{BC}$ and placed probes with appropriate tips for tympanometry.

\section{Follow-Up Protocol}

The audiologist reviewed the stored and forwarded video-otoscopy images and pure-tone audiometry and tympanometry findings. Findings of suspected MED cases were emailed to the otolaryngologists for review, who then provided appropriate recommendations within a one-week period. Follow-up tele-audiological surveillance was conducted at the individuals' homes after the recommendations were followed.

Those requiring surgical intervention were recommended to visit the tertiary care hospital. The audiologist conducted informal hearing aid trials through videoconferencing with assistance from the CBRWs. Hearing aids with suitable gain (similar to those provided by the government) were used for the trial. Hearing aid prescriptions and audiological test results were provided to individuals who presented with sensorineural hearing loss of $>40 \mathrm{~dB} \mathrm{HL}$ in their better ear; these individuals were referred to the district hospital to procure free government-issued hearing aids.

\section{In-Person Audiological Surveillance}

In-person audiological surveillance was conducted in Thiruvannamalai district residents at three camps, two of which were at a nongovernmental organization and the third at a local public school.

\section{Test Location and Testing Setup}

Noise levels in the test room were monitored by audiologists using BOSCH-iV (Robert Bosch Engineering and Business Solutions Limited) to ensure ambient noise levels of $\leq 40 \mathrm{dBA}$. Video-otoscopy, pure-tone audiometry, and tympanometry were performed by the audiologist following standard testing procedures. Images were viewed in real time, and recommendations were provided directly to the beneficiaries. Individuals with impacted cerumen and suspected MEDs were referred for otolaryngologist consultations at the local clinic or district hospital. Pure-tone audiometry and tympanometry were performed on all individuals, including those with the presence of cerumen at baseline measurement.

\section{Follow-Up Protocol for In-Person Audiological Surveillance}

The CBRWs conducted follow-ups through periodical phone calls to review whether individuals had sought an otolaryngologist's opinion. Follow-up audiological surveillance was conducted at subsequent camps in individuals who received otolaryngologist consults and followed recommendations. Hearing aid trials were conducted at campsites using the same protocol as that used in the tele-audiological surveillance group.

\section{Analysis}

The coverage, follow-up rate for intervention (medical/ surgical/audiological), and cost outcomes from the provider's perspective were analyzed and compared between the tele-audiological and in-person audiological surveillance groups.

\section{RESULTS}

I $\mathrm{n}$ Cuddalore district, video-otoscopy, pure-tone audiometry, and tympanometry were performed on 30 individuals. Tympanometry was not feasible in two individuals because of bilateral ear discharge. In Thiruvannamalai district, video-otoscopy, pure-tone audiometry, and tympanometry were performed on 25 individuals. Among these individuals, the cleft types included bilateral (16 cases) and unilateral (24 cases) CLPs, craniofacial clefts (2 cases), and cleft palate (13 cases).

\section{Coverage}

In Cuddalore district, 30 of the 44 individuals (23 males, 7 females; aged between 9 and 28 years) underwent tele-audiological surveillance. Scheduled appointments for home testing were completed in 8 days, and campsite testing was completed at three camps. Overall, $68 \%$ coverage was achieved for tele-audiological surveillance.

In Thiruvannamalai district, 25 of the 65 individuals (12 males, 13 females; aged between 6 and 22 years) underwent in-person audiological surveillance at three camps organized during the study period. Overall, $38 \%$ coverage was achieved. Figure 2 summarizes the coverage obtained with the two models of testing.

\section{Follow-Up Compliance}

\section{Tele-Audiological Surveillance}

In Cuddalore district, 18 of the 30 individuals were required to follow-up for treatment of cerumen, MED, or hearing aid fitting. Figure 3 outlines the outcomes of video-otoscopy and hearing evaluations, recommendations provided, and the follow-up compliance in this group.

Nine of the 13 individuals with impacted cerumen were recommended ear drops (Soliwax; Nulife Pharmaceuticles, India) for cerumen management, and all were 


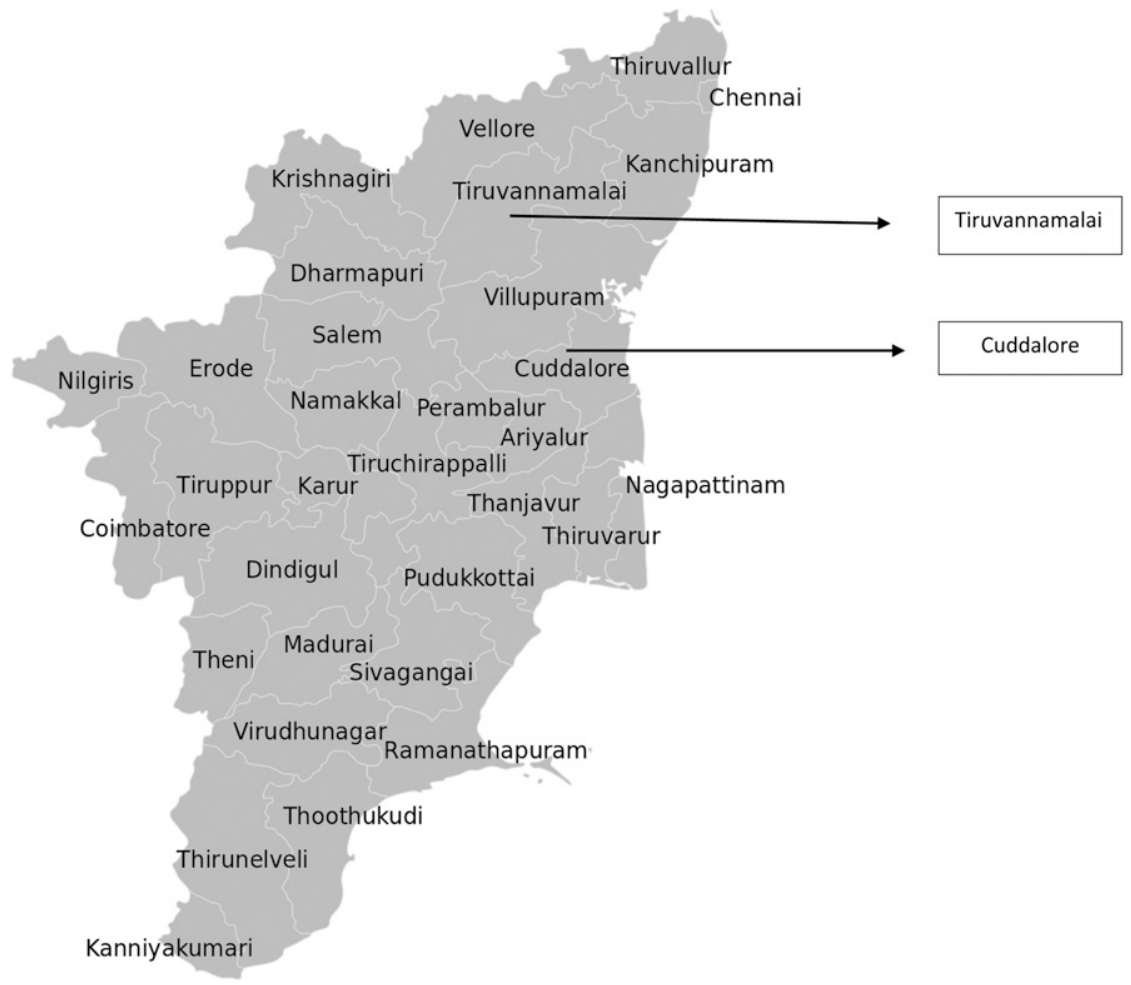

Figure 1. Map of Tamil Nadu depicting the two districts under the study.

compliant and reported for follow-up testing. Four individuals with limited internet access underwent homebased testing at the home of the nearest beneficiary undergoing follow-up using tele-audiological testing. All individuals underwent repeat video-otoscopy, pure-tone audiometry, and tympanometry. The results of video-otoscopy revealed that all nine individuals had intact bilateral TMs. The findings of bilateral normal hearing sensitivity obtained at initial testing were reconfirmed during follow-up testing in all these individuals. In addition, all nine individuals who initially had bilateral B-type tympanograms without reflexes showed bilateral A-type tympanograms with reflexes at follow-up testing, suggestive of normal middle ear function.

Two of the four individuals diagnosed with MED were recommended for surgery; of these, one individual underwent myringotomy with grommet insertion at the
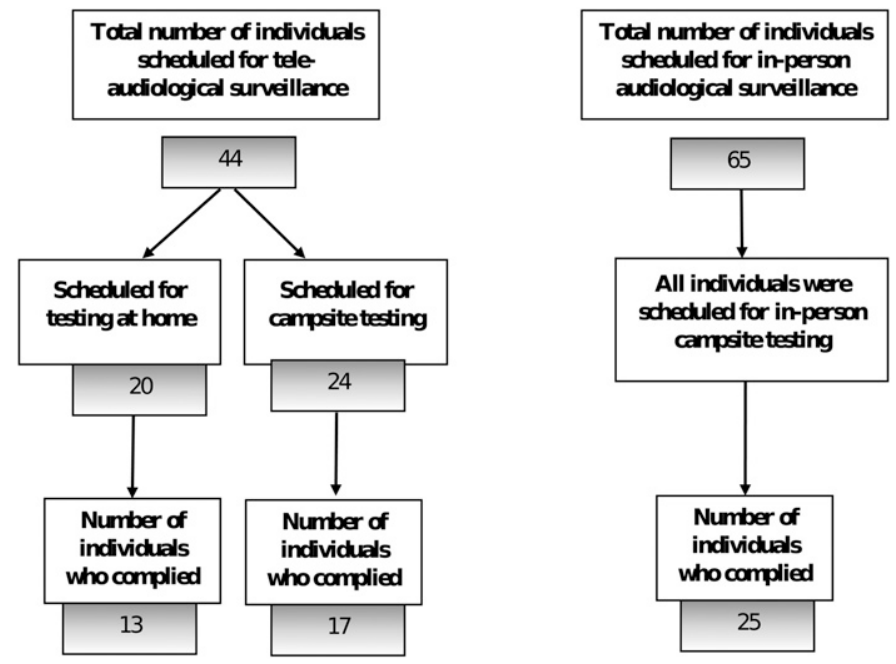

Figure 2. Coverage in tele- and in-person audiological surveillance groups. 


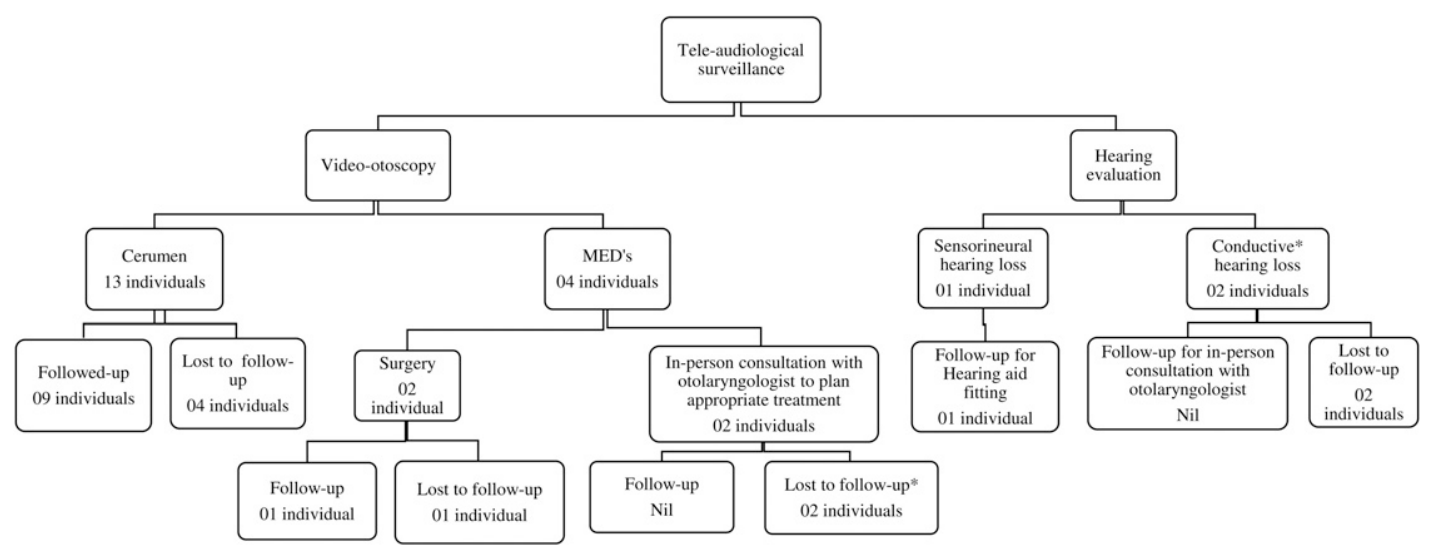

*These two individuals are accounted under MEDs

Figure 3. Follow-up compliance for tele-audiological surveillance.

tertiary care hospital. The other two individuals with unilateral conductive hearing loss, who were recommended for in-person otolaryngologist consultation for treatment plans, did not comply.

One individual with bilateral moderate sensorineural hearing loss was provided a home-based telehearing aid trial facilitated by CBRWs, who procured governmentissued hearing aids from the district hospital.

Overall, 11 of 18 individuals (61\%) were compliant and underwent follow-up tele-audiological surveillance, with a minimum follow-up period of 1 month and maximum follow-up period of 2.5 months from initial diagnosis.

\section{In-Person Audiological Surveillance}

In Thiruvannamalai district, 21 of 25 individuals were required to follow-up for treatment of cerumen, MED, or hearing aid fitting. Figure 4 outlines the outcomes of video-otoscopy and hearing evaluations, recommendations provided, and the follow-up compliance in the in-person audiological surveillance group.

Four of the 11 individuals with impacted cerumen who were recommended ear drops (Soliwax) were compliant and reported for follow-up testing, which was conducted in camps. All individuals underwent repeat video-otoscopy, pure-tone audiometry, and tympanometry. The results of video-otoscopy revealed that all four individuals had intact bilateral TMs. The findings of bilateral normal hearing sensitivity obtained at initial testing were reconfirmed at follow-up testing. Three individuals with bilateral B-type tympanograms and one individual with unilateral B-type tympanograms, both without reflexes at initial tympanometry, showed bilateral A-type tympanograms with reflexes at follow-up testing, suggestive of normal middle ear function. None of the seven individuals with suspected MED received consultations with the local otolaryngologist for treatment plans. Hence, follow-up audiological surveillance could not be performed. One individual

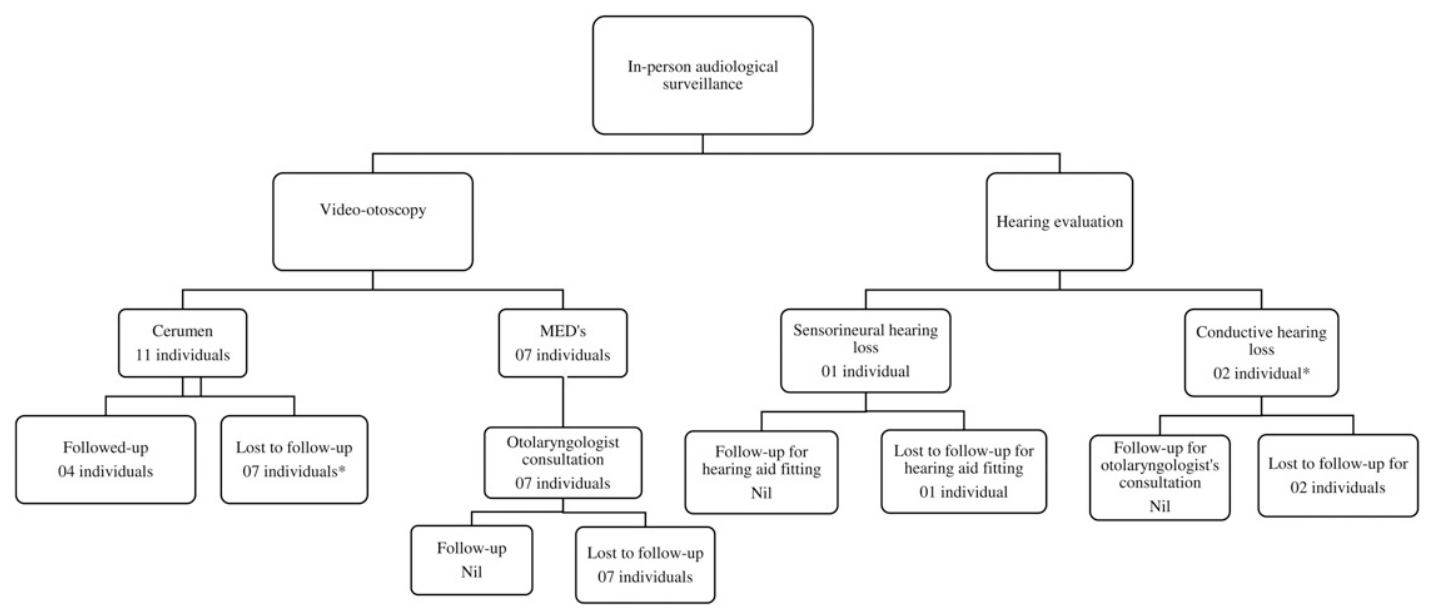

*These two individuals are accounted under those with cerumen who were lost to follow-up

Figure 4. Follow-up compliance for in-person audiological surveillance. 
with bilateral moderate sensorineural hearing loss did not comply with the audiologist's recommendation of hearing aid trial at the district or tertiary care hospital, and two individuals, among those with unilateral mild conductive hearing loss, did not comply with the local otolaryngologist's recommendation of follow-up, including cerumen removal or MED evaluation. Therefore, 4 of 21 individuals (19\%) were compliant and available at follow-up through in-person audiological surveillance, with a minimum duration of 2 months and maximum duration of 5.7 months from initial diagnosis.

Tele-audiological testing enabled diagnoses and management plans at the community level. With the treatment provided at the community level, follow-up compliance was better (61\%) than in-person audiological surveillance (19\%). For example, one individual with bilateral sensorineural hearing loss, who underwent home-based telehearing aid fitting, obtained hearing aids from the district hospital at the audiologist's recommendation. Similarly, $69 \%$ of individuals underwent treatment using ear drops for cerumen management. However, individuals requiring surgery or in-person otolaryngologist consultations regarding treatment plans had a loss to follow-up rate of $75 \%$. Despite a higher overall follow-up compliance rate in individuals who underwent teletesting, this was only achieved through services provided at the community level. Similarly, in Thiruvannamalai district, referrals were made to local otolaryngologists for further diagnoses and treatment plans; however, because of poor follow-up, the rate

Table 1. Costs Considered in Each Input Category of the Two Alternative Models of Audiological Surveillance

\begin{tabular}{|c|c|c|c|c|c|c|c|}
\hline \multicolumn{8}{|c|}{ Cost Estimation of Tele-Audiological Surveillance } \\
\hline \multicolumn{5}{|c|}{ Capital Cost } & \multicolumn{3}{|c|}{ Recurrent Cost } \\
\hline \multicolumn{8}{|c|}{ Direct medical cost } \\
\hline S. No. & Heads & Quantity & $\begin{array}{l}\text { Unit cost } \\
(\cup S \$)\end{array}$ & $\begin{array}{c}\text { Actual cost } \\
\text { (US\$) }\end{array}$ & $\begin{array}{l}\text { Number/ } \\
\text { Frequency }\end{array}$ & $\begin{array}{l}\text { Unit cost } \\
\text { (US\$) }\end{array}$ & $\begin{array}{l}\text { Actual cost } \\
\quad(\text { US } \$)\end{array}$ \\
\hline \multicolumn{8}{|c|}{ Equipment } \\
\hline 1 & Audiometer \& tympanometer & 1 & $\$ 5,530$ & $\$ 5,530$ & & & \\
\hline 2 & Video-otoscope & 1 & $\$ 833$ & $\$ 833$ & & & \\
\hline 3 & Laptop computers for teletesting & 2 & $\$ 454$ & $\$ 908$ & & & \\
\hline 4 & Mobile phone used for internet in community & 1 & $\$ 91$ & $\$ 91$ & & & \\
\hline 5 & Internet dongle & 1 & $\$ 15$ & $\$ 15$ & & & \\
\hline \multicolumn{8}{|c|}{ Direct non-medical cost } \\
\hline 1 & CBRW's travel charges & & & & 8 days & $\begin{array}{c}\$ 3 \text { petrol } \\
\text { charge/day }\end{array}$ & $\$ 24$ \\
\hline 2 & Audiologist salary & & & & 22 hours & $\begin{array}{l}\text { \$6 salary } \\
\text { cost/hour }\end{array}$ & $\$ 132$ \\
\hline 3 & Internet recharge for mobile phone data & & & & 2 times & \$6/recharge & $\$ 12$ \\
\hline 4 & Internet recharge for audiologist's internet dongle & & & & 3 times & $\$ 12 /$ recharge & $\$ 36$ \\
\hline 5 & Travel cost reimbursed for CBRW & & & & $\begin{array}{l}2 \text { CBRWs } \\
3 \text { camps }\end{array}$ & - & $\$ 55$ \\
\hline \multicolumn{8}{|c|}{ Direct medical cost } \\
\hline \multicolumn{8}{|c|}{ Consumables } \\
\hline 1 & Alcohol swabs & & & & 2 nos. & $\$ 4$ & $\$ 7$ \\
\hline 2 & Cotton bundle & & & & 1 nos. & $\$ 3$ & $\$ 3$ \\
\hline 3 & Sterilium bottle & & & & 1 nos. & $\$ 5$ & $\$ 5$ \\
\hline \multicolumn{8}{|c|}{ Cost estimation of in-person audiological surveillance } \\
\hline \multicolumn{8}{|c|}{ Direct medical cost } \\
\hline \multicolumn{8}{|c|}{ Equipment } \\
\hline 1 & Audiometer \& tympanometer & 1 & $\$ 5,530$ & $\$ 5,530$ & & & \\
\hline 2 & Video-otoscope & 1 & $\$ 833$ & $\$ 833$ & & & \\
\hline \multicolumn{8}{|c|}{ Direct non-medical cost } \\
\hline 1 & Audiologist salary & & & & 3 camps & $\$ 15$ & $\$ 45$ \\
\hline 2 & Audiologist travel time cost & & & & 36 hours & $\$ 74$ & $\$ 222$ \\
\hline 3 & Travel cost to campsite & & & & 3 camps & - & $\$ 222$ \\
\hline 4 & Travel cost reimbursed for CBRW & & & & $\begin{array}{l}2 \text { CBRWs } \\
3 \text { camps }\end{array}$ & - & $\$ 25$ \\
\hline \multicolumn{7}{|c|}{ Direct medical cost } & Consumables \\
\hline 1 & Cotton bundle & & & & 1 & $\$ 3$ & $\$ 3$ \\
\hline 2 & Alcohol swabs & & & & 2 & $\$ 4$ & $\$ 7$ \\
\hline 3 & Sterilium bottle & & & & 1 & $\$ 5$ & $\$ 5$ \\
\hline
\end{tabular}


for seeking diagnoses and treatment plans from local otolaryngologists was poor (19\%).

\section{Cost Outcomes of Tele- and In-Person Audiological Surveillance}

Cost outcomes were estimated for the two models as per the WHO framework for cost guideline (Creese and Parker, 1994) from the provider's perspective. The study was conducted from June 2017 to December 2017. Costs were expressed in USD, based on the average conversion rate of Indian Rupees in April 2018, and approximated to the nearest whole number (Table 1).

In each model, capital costs included those of the audiometer, tympanometer, and video-otoscope, whereas recurrent costs included those of the audiologists' salaries, including consumables such as cotton bundles (Jaycot, India), Uniclean alcohol wipes (Manward Health Care Products Pvt Ltds, India), and Sterillium ${ }^{\circledR}$ hand sanitizer (Hartmann, Germany). In addition, travel costs of the CBRWs to testing sites were included, as these were reimbursed by Transforming Faces, Canada. With regard to the tele-audiological surveillance, capital costs included those of laptop and internet dongles and recurrent costs included those of internet recharge and the CBRWs' mobile phones (used for internet access in the community). With regard to the in-person audiological surveillance, recurrent costs included those of the audiologists' travels, including travel time. With both methods, cost of space was not included, as test locations did not incur any rental costs.

Equipment costs were based on purchase invoice records. Travel costs were assigned based on taxi bills and bus tickets. Recurrent costs incurred by the CBRWs, such as gas bills and mobile data recharge costs, were obtained directly from each CBRW. Audiologists' salaries and time costs were allocated proportional to the time spent on work, which was determined from the time sheets. Total input cost based on those of itemized activities within each audiological surveillance model was obtained to determine the total amount spent on the respective model. Total costs incurred through tele-audiological and in-person audiological surveillance is shown in Table 2.

Table 2. Cost Incurred through Tele-Audiological and In-Person Audiological Surveillance

\begin{tabular}{lll}
\hline & Tele- & In-Person \\
& $\begin{array}{l}\text { Audiological } \\
\text { Surveillance }\end{array}$ & $\begin{array}{l}\text { Audiological } \\
\text { Surveillance }\end{array}$ \\
\hline Capital cost & $\$ 7,377$ & $\$ 6,363$ \\
Recurrent cost & $\$ 274$ & $\$ 529$ \\
Total & $\$ 7,651$ & $\$ 6,892$ \\
Cost per individual tested & $\$ 191$ & $\$ 238$ \\
\hline
\end{tabular}

The capital cost of tele-audiological surveillance was higher because of the need for laptops and internet dongles to support tele-audiological testing. However, the recurrent cost of in-person audiological surveillance was higher because of the audiologists' travel costs.

The total cost per model was divided by the total number of individuals tested in that model, resulting in the cost-per-unit outcome. Thirty individuals underwent initial tele-audiological surveillance, of which, ten individuals underwent post-treatment follow-up testing. The cost per individual tested through tele-audiological surveillance was USD 191. Of the 25 individuals who underwent initial in-person audiological surveillance, four were available at post-treatment follow-up testing, and the cost per in-person audiological surveillance was USD 238. Thus, the amount saved per individual undergoing tele-audiological surveillance versus in-person audiological surveillance was USD 47. The lower cost reflects higher coverage and follow-up compliance rates in the tele-audiological surveillance group.

\section{DISCUSSION}

$\mathrm{T}$ Tele-audiological surveillance provided the flexibility to conduct testing either at home or at the campsite, depending on internet availability, as the audiologist was not required to be available at the test site. Home-based testing was preferred based on our previous experiences of poor follow-up rates for in-person camps. However, the results of this study show that the coverage for tele-audiological surveillance was similar irrespective of testing site.

Even though campsite testing is common to both arms of this study, tele-audiological testing at campsites was easier to schedule as the patient's availability was the main consideration. For in-person campsite testing, camp dates and timings were determined based on the mutual convenience of both the audiologist and patient, which was more challenging.

\section{Follow-Up Compliance}

In the present study, the results revealed a higher follow-up compliance rate in individuals undergoing tele-audiological tests, in agreement with those reported in previous studies (Elliott et al, 2010; Dharmar et al, 2016; Ramkumar et al, 2018). Elliot et al (2010), reported an increase in follow-up compliance rates (59\%) in children between 0 and 16 years, attending schools and day care centers in Queensland, Australia. Results were evaluated based on tele-pure-tone audiometry, tympanometry, and video-otoscopy testing conducted in a mobile van over a period of 6 months. The compliance rate of $59 \%$ within the six-month period was equal to the rate of $59 \%$ obtained within a one-year period before 
the implementation of teletesting, indicating that a shorter time was required to achieve the same coverage. Dharmar et al (2016), reported a 100\% follow-up compliance rate in 22 infants from a rural community in Northern California, USA, undergoing telediagnostic auditory brainstem response (ABR); however, they reported a loss to follow-up rate of $22 \%$ for in-person diagnostic ABR 2 years before implementing telediagnostic ABR. Similarly, Ramkumar et al (2018) reported an $86 \%$ follow-up compliance rate for tele-ABR conducted in children younger than 5 years who failed the distortion product otoacoustic emission screening performed at the homes of children in a rural community in Tamil Nadu, India.

In this study, individuals of a district were allotted to one model of audiological surveillance in a purposive manner. Because this was a community trial, randomization was not feasible. The CBRWs in Cuddalore district were trained in video-otoscopy and were experienced in using the technology, and, hence, this district was assigned to receive tele-audiological surveillance. Because of the considerations of geographical proximity, the CBRW from one district could not be assigned to another district. Therefore, the outcomes of follow-up may be somewhat influenced by the access barriers, enthusiasm of the CBRW, and internet penetration inherent to a particular district.

\section{Cost Outcomes of Tele-Audiological and In-Person Audiological Surveillance}

Our results indicated that the overall coverage and follow-up compliance rates of tele-audiological surveillance were better than those obtained with in-person audiological surveillance. Tele-audiological surveillance resulted in savings for the provider, with a shorter timeline between the diagnoses and follow-ups than that with in-person audiological surveillance.

Several researchers have reported that telepractices are cost-effective and cost-saving in disciplines such as teleradiology (Roine et al, 2001), telecardiology (Kifle et al, 2006), and teleconsultations for allied health assessments, including speech and language, and occupational and physical therapy (Hassall et al, 2003). However, there is limited literature to clarify the comparison of costs between telemodels and in-person models for service delivery. A study including children with hearing loss who underwent teleintervention in Utah, USA, reported costsavings between USD 56,280 and USD 6,970 compared with the in-person intervention over a 24-month period (Blaiser et al, 2013). Ramkumar et al (2018), reported the cost outcomes of community-based hearing screening programs integrated with remote diagnostic $A B R$ via tele-audiology. Their results indicated that the cost per followed-up participant using telediagnostic $\mathrm{ABR}$ and per child identified with hearing loss was
Rs 1,59,930 (USD 2,400) and Rs 6,07,734 (USD 9,120), respectively. Furthermore, in Bangladesh, the cost of fitting hearing aids in the rural community was cheaper by BDT 7,385 than that of fitting hearing aids at private health centers (Ekman and Borg, 2017). Therefore, the cost-savings of outreach services to the community is evident. The magnitude of cost-saving is likely to increase in outreach services targeting larger populations.

\section{CONCLUSION}

$\Upsilon$ he results from this study suggested that the cov1 erage for tele-audiological surveillance was better than that for in-person audiological surveillance. The overall follow-up compliance rate with tele-audiological surveillance was better than that with in-person audiological surveillance. With tele-audiological surveillance, the follow-up compliance rates were higher only when management was provided locally at the community level (e.g., cerumen management and hearing aid fitting). The cost per individual tested through tele-audiological surveillance was lower than that through in-person audiological surveillance. Therefore, it would be beneficial to program planners to implement tele-audiological surveillance to achieve higher success rates in identifying and treating CLP patients with MED in rural locations.

\section{REFERENCES}

American Cleft-Palate Craniofacial Association. (2009) Parameters for evaluation and treatment of patients with cleft lip/palate or other craniofacial anomalies. American Cleft Palate-Craniofacial Association. March, 1993. Cleft Palate Craniofac J 30(S1-16, Suppl).

Blaiser KM, Behl D, Callow-Heusser C, White KR. (2013) Measuring costs and outcomes of tele-intervention when serving families of children who are deaf/hard-of-hearing. Int J Telerehabil 5(2):3-10.

Bluestone CD, Paradise JL, Beery QC, Wittel R. (1972) Certain effects of cleft palate repair on eustachian tube function. Cleft Palate $J$ 9:183-193.

Brennan-Jones CG, Eikelboom RH, Bennett RJ, Tao KF, Swanepoel W. (2018) Asynchronous interpretation of manual and automated audiometry: agreement and reliability. $J$ Telemed Telecare 24(1):37-43.

Creese A, Parker D. (1994) Cost Analysis in Primary Health Care: A Training Manual for Programme Managers. Geneva, Switzerland: WHO Publishers Centre.

Dharmar M, Simon A, Sadorra C, Friedland G, Sherwood J, Morrow H, Deines D, Nickell D, Lucatorta D, Marcin JP. (2016) Reducing loss to follow-up with tele-audiology diagnostic evaluations. Telemed J E Health 22(2):159-164.

D’Mello J, Kumar S. (2007) Audiological findings in cleft palate patients attending speech camp. Indian J Med Res 125(6): $777-782$. 
Ekman B, Borg J. (2017) Provision of hearing aids to children in Bangladesh: costs and cost-effectiveness of a community-based and a centre-based approach. Disabil Rehabil Assist Technol 12(6):625-630.

Elliott G, Smith AC, Bensink ME, Brown C, Stewart C, Perry C, Scuffham P. (2010) The feasibility of a community-based mobile telehealth screening service for Aboriginal and Torres Strait Islander children in Australia. Telemed J E Health 16(9):950-956.

Feniman MR, Souza AG, Jorge JC, Lauris JR. (2008) Otoscopic and tympanometric findings in infants with cleft lip and palate. Braz J Otorhinolaryngol 74(2):248-252.

Flynn T, Möller C, Jönsson R, Lohmander A. (2009) The high prevalence of otitis media with effusion in children with cleft lip and palate as compared to children without clefts. Int J Pediatr Otorhinolaryngol 73(10):1441-1446.

Hassall S, Wootton R, Guilfoyle C. (2003) The cost of allied health assessments delivered by videoconference to a residential facility for elderly people. J Telemed Telecare 9(4):234-237.

Kifle M, Mbarika VWA, Datta P. (2006) Interplay of cost and adoption of tele-medicine in Sub-Saharan Africa: the case of telecardiology in Ethiopia. Inf Syst Front 8(3):211-223.

Kuehn DP, Moller KT. (2000) Speech and language issues in the cleft palate population: the state of the art. Cleft PalateCraniofacial J 37(4):348.
Millard T, Richman LC. (2001) Different cleft conditions, facial appearance, and speech: relationship to psychological variables. Cleft Palate-Craniofacial $J$ 38(1):68-75.

Pearce W, Ching TYC, Dillon H. (2009) Brief communications hearing services using tele-audiology to remote areas. Aust New Zealand J Audiol 31(2):96-100.

Ramkumar V, John KR, Selvakumar K, Vanaja CS, Nagarajan R, Hall JW. (2018) Cost and outcome of a community-based paediatric hearing screening programme in rural India with application of tele-audiology for follow-up diagnostic hearing assessment. Int $J$ Audiol 57(6):407-414.

Roine R, Ohinmaa A, Hailey D. (2001) Assessing telemedicine: a systematic review of the literature. Can Med Assoc $J$ 165(6): 765-771.

Strauss RP, Broder H, Helms RW. (1988) Perceptions of appearance and speech by adolescent patients with cleft lip and palate and by their parents. Cleft Palate $J$ 25(4):335-342.

Subramaniam V, Manuprasad S, Kallikkadan HH, Vijay Kumar K. (2015) Otological and audiological manifestations in cleft lip and cleft palate children: a clinical study. Int J Otorhinolaryngol Head Neck Surg 1(1):7-10.

World Health Organization (WHO). (1991) Report of the Informal Working Group on Prevention of Deafness and Hearing Impairment Programme Planning, Geneva, 18-21 June 1991. Geneva, Switzerland: World Health Organization. 